\title{
Manejo de hiperkalemia en la emergencia de un hospital general
}

\author{
Management of hyperkalemia at the emergency service of a \\ general hospital
}

\author{
Percy Herrera Añazco ${ }^{1,3, a}$, Melisa Palacios Guillén ${ }^{4,5, a}$, David Chipayo Gonzales ${ }^{1, b}$, \\ Marievelyn Barrenachea Gómez ${ }^{2, \mathrm{c}}$, Manuela Silveira Chau ${ }^{6, \mathrm{~d}}$ \\ ${ }^{1}$ Servicio de Nefrología, Hospital Nacional 2 de Mayo, Lima, Perú. \\ ${ }^{2}$ Facultad de Medicina, Universidad Científica del Sur, Lima, Perú. \\ ${ }^{3}$ Facultad de Medicina, Universidad Nacional de Piura, Piura, Perú. \\ ${ }^{4}$ Servicio de Nefrología, Hospital Daniel Alcides Carrión, Callao, Perú. \\ ${ }^{5}$ Facultad de Medicina, Universidad Nacional Mayor de San Marcos, Lima, Perú. \\ ${ }^{6}$ Clínica Internacional, Lima, Perú. \\ ${ }^{a}$ Médico nefrólogo; ${ }^{b}$ Médico residente de nefrología; ${ }^{\circ}$ Estudiante de medicina; ${ }^{d}$ Médico cirujano.
}

An Fac med. 2014;75(2):201-2 / doi: http://dx.doi.org/10.15381/anales.v75i2.8392

\section{SEÑOR EDITOR}

La hiperkalemia (HK) es una patología frecuente y potencialmente fatal en los servicios de emergencia ${ }^{(1)}$. Debido a ello, es necesaria una atención oportuna y adecuada, la misma que, en el contexto de una estructura de atención limitada por procesos burocráticos engorrosos y sobrepoblación de los servicios de emergencia -como se presenta en los hospitales del $\mathrm{Mi}$ nisterio de Salud (MINSA)-, se puede traducir en retraso en el diagnóstico y en el inicio de terapia, así como en indicaciones terapéuticas deficientes con complicaciones que deriven en muerte de pacientes ${ }^{(2)}$.

Por ello, llevamos a cabo un estudio retrospectivo de todos los pacientes con potasio $(\mathrm{K})$ mayor de $6 \mathrm{meq} / \mathrm{L}$ que acudieron al servicio de emergencia del Hospital Nacional 2 de Mayo (HN2M) entre los meses de septiembre de 2011 y febrero de 2012, excluyéndose a todos los pacientes trasferidos de otros hospitales que, teniendo diagnóstico de hiperkalemia $(\mathrm{HK})$, ya habían recibido terapia en su hospital de referencia.

Evaluamos el tiempo de diagnostico de HK definido como 'El tiempo entre la entrega de la hoja de atención y la hora de llegada del resultado de electrolitos'; el tiempo de inicio de terapia definido como 'el tiempo entre el diagnóstico de HK y la hora de administración del primer fármaco asociado al manejo de la misma' escrito en el cárdex de enfermería. Así mismo, describimos el tipo de terapia empleada y se la comparó con las recomendaciones propuestas por la United Kingdom (UK) Renal Association (RA) en su Clinical Practice Guidelines Treatment of Acute Hyperkalaemia in Adults ${ }^{(3)}$. Por último, se determinó el tiempo en que se solicitó el primer control de $\mathrm{K}$ luego del inicio de la terapia.

Se estudió 54 casos, con una edad media de 64,9 $\pm 13,8$ años, de los cuales 31 pacientes fueron varones. $\mathrm{La}$ media de tiempo para el diagnóstico de HK fue 3,27 horas (DE: 2,16) y para el inicio de tratamiento 2,39 horas (DE:
3,13). El primer control de electrolitos luego del inicio del tratamiento demoró más de 24 horas en 16,7\% de los casos; los demás tuvieron un tiempo promedio de $8,74 \pm 5,85$ horas.

De los 54 pacientes, el 92,6\% recibió tratamiento; a 94\% de ellos se administró agonista B-adrenérgico (fenoterol), aunque con dosis o forma correcta solo en $21,3 \%$; $34 \%$ recibió insulina, pero solo $52,9 \%$ lo hizo con dosis o frecuencia correcta; a $96 \%$ se administró gluconato de calcio, aunque del $50 \%$ que tenía electrocardiograma solo $35,4 \%$ tenía cambios electrocardiográficos propios de $\mathrm{HK}{ }^{(3)}$. Finalmente, aunque $66 \%$ recibió bicarbonato, solo $60,6 \%$ tenía acidosis (definida como bicarbonato menor a $18 \mathrm{meq} / \mathrm{litro}$ ).

En nuestros pacientes, el tiempo desde el diagnóstico de HK hasta el inicio de terapéutica fue cerca de 6 horas. Acker encontró un tiempo promedio de 2,2 horas entre la llegada a emergencia y la administración de la primera droga ${ }^{(4)}$. Mientras que Freeman halló 
que el tiempo promedio hasta la primera intervención en el paciente con hiperkalemia fue 117 minutos ${ }^{(5)}$. Por otro lado, a pesar de que en promedio se recomienda un primer control de potasio a la hora del inicio de la terapéutica ${ }^{(3)}$, en nuestros pacientes trascurrieron cerca de 9 horas hasta el primer control.

Hay muchas razones que podrían explicar el retraso del diagnóstico e inicio de la terapia: el tiempo que demora sacar la historia clínica, la compra de medicamentos y la solicitud de ayuda con la asistente social, en algunos casos, pueden ser parte de las causas. Así mismo, la abundante carga asistencial en los turnos de guardia para poco personal médico y la deficiente capacidad de algunos colegas para priorizar la atención, entre otras razones. Sea la razón que fuere, es claro es que hay un excesivo tiempo transcurrido entre la llegada del paciente, la prescripción de la primera droga y el primer control de $\mathrm{K}$ comparado con experiencias en otros centros asistenciales.

Con respecto al tratamiento, las guías de la UK Renal sugieren que las nebulizaciones con beta agonistas y la insulina son las intervenciones de primera línea y que su efecto es mejor si se usa en combinación ${ }^{(3)}$. En nuestro estudio, tanto los beta agonistas y la insulina fueron medicamentos frecuentemente usados; sin embargo, la dosis y la frecuencia no fueron las óptimas. El gluconato de calcio fue el fármaco más usado entre nuestros pacientes, a pesar de que se ha descrito que solo hay estudios anecdóticos del uso de sales de calcio como estabilizador de membrana; y, aunque no hay evidencia de su utilidad, existe una plausibilidad biológica que aconseja su uso en caso de cambios electrocardiográficos sugestivos de hiperkalemia ${ }^{(6)}$, lo que en nuestro caso ocurrió en menos de la mitad de los EKG tomados. Por otro lado, si bien un poco más de la mitad de nuestros pacientes recibió bicarbonato, no todos tenían acidosis metabólica, a pesar de que no se ha encontrado evidencia de la utilidad del bicarbonato en los pacientes sin acidosis ${ }^{(3,6)}$.

Nuestro estudio presenta limitaciones importantes, como el hecho de que al ser unicéntrico no es generalizable. Así mismo, al ser observacional, no revela causalidad de los hallazgos descritos. Sin embargo, dado los problemas burocráticos y de carga asistencial en los diferentes hospitales del MINSA, no es ilógico pensar que la misma situación se puede presentar en el resto de hospitales. Por otro lado, al comunicar los hallazgos electrocardiográficos descritos en la historia clínica pudieron existir casos con cambios en el EKG no advertidos por el médico tratante. De igual forma debimos confiar en que las horas consignadas en la historia clínica fueron las reales.

En conclusión, a pesar de que casi todos los casos de HK tuvieron tratamiento, este fue deficiente. Así mismo, el tiempo de diagnóstico y de inicio de tratamiento fue prolongado. En ese sentido, es necesario evaluar lo procesos de atención en las emergencias para conseguir una atención más eficiente en términos de calidad, en beneficio de nuestros pacientes.

\section{AGRADECIMIENTOS}

Los autores agradecen al doctor Henry Yupanqui Caldera jefe del servicio de emergencia del HN2M, por su apoyo al desarrollo del trabajo.

\section{REFERENCIAS BIBLIOGRÁFICAS}

1. Shingarev R, Allon M. A physiologic-based approach to the treatment of acute hyperkalemia.Am J Kidney Dis.2010;56(3):578-84. doi: http://dx.doi. org/10.1053/j.ajkd.2010.03.014

2. Weisberg LS. Management of severe hyperkalemia. Crit Care Med. 2008;36(12):3246-51.doi: http:// dx.doi.org/10.1097/CCM.0b013e31818f222b

3. UK Renal Association. Clinical Practice Guidelines. Treatment of Acute Hyperkalaemia in Adults.2012. Disponible en: http://www.renal.org/Libraries/ Guidelines/Treatment_of_Acute_Hyperkalaemia_in_Adults_-_FINAL_VERSION_JULY_2012. sflb.ashx

4. Acker CG, Johnson JP, Palevsky PM, Greenberg A. Hyperkalemia in hospitalized patients. Causes, adequacy of treatment, and results of an attempt to improve physician compliance with published therapy guidelines. Arch Intern Med. 1998;158(8):917-24. doi: http://dx.doi.org/10.1001/ archinte.158.8.917

5. Freeman K, Feldman JA, Mitchell P, Donovan J, Dyer KS, Eliseo L, White LF, Temin ES. Effects of presentation and electrocardiogram on time to treatment of hyperkalemia. Acad Emerg Med.2008;15(3):239-49. doi: http://dx.doi. org/10.1111/j.1553-2712.2008.00058.x

6. Mahoney BA, Smith WAD, Lo D, Tsoi K, Tonelli $M$, Clase C. Emergency interventions for hyperkalaemia (Review). Cochrane Database Syst Rev. 2005;(2):CD003235. doi: http://dx.doi. org/10.1002/14651858.CD003235.pub2

Los autores declaran no existir conflictos de interés.

\section{Correspondencia:}

Dr. Percy Herrera Añazco

Olavegoya 1879 dpto 701 Jesús Maria Lima 11

Correo electrónico: silamud@hotmail.com 